\title{
DISPOSABLE MICRODEVICES FOR DNA ANALYSIS AND CELL SORTING
}

\author{
Hou-pu Chou, Charles Spence, Anne Fu, Axel Scherer and Stephen Quake* \\ Dept of Applied Physics, California Institute of Technology \\ Pasadena, CA 91125
}

\begin{abstract}
We have developed microfabricated devices to size and sort microscopic objects, based on measurement of fluorescent properties. With these devices, we have demonstrated sizing and sorting on various biological entities, ranging from $E$. Coli cells to single molecules of DNA. The microfabricated devices have several advantages over macroscopic systems, including size, cost and sensitivity. For example, the detection volume for our devices is 375 femtoliters, more than an order of magnitude smaller than what has been achieved with flow cytometry.
\end{abstract}

\section{INTRODUCTION}

Traditionally, biologists have measured the size of molecules indirectly - they first measure mobility with gel electrophoresis, and then infer the size from the mobility. This method is powerful, yet has some drawbacks. For medium to large DNA molecules the resolution is limited to approximately $10 \%$. Gel electrophoresis is time consuming; it generally takes at least an hour to run the gel, not including the set up time to cast the gel. Furthermore, for large molecules the procedure fails. This has been alleviated to some extent by the development of pulsed field gel electrophoresis, but running times can be days.

With the development of high affinity intercalating DNA stains, it has become possible to directly measure the length of single molecules by quantitating fluorescence. This in principle allows the measurement of extremely long DNA molecules; in fact, the longer the molecule, the better the signal. Other groups have used this technique with traditional methods in flow cytometry to measure length distributions of DNA molecules.[1][2]

We have developed microfabricated devices to size and sort microscopic objects based on their fluorescent properties. The devices are fabricated out of silicone elastomer, using a replica method. Negative master devices are fabricated from silicon wafers using standard processing techniques. Liquid elastomer is poured on the wafer and cured in an oven. The resulting device can be peeled off of the wafer and will bond hermetically to glass. This is a simple and cheap process, allowing the devices to be disposable while the master wafers are recycled indefinitely. This fabrication technique was first suggested by Austin's group at Princeton [3], building on work by the Whitesides group at Harvard. [4]

Previously, we have shown that these devices could be used to size a homogenous solution of $48 \mathrm{kbp}$ DNA with $10 \%$ resolution.[5] We have now extended those results to demonstrate sizing of DNA molecules ranging in length from $2 \mathrm{kbp}$ to $200 \mathrm{kbp}$ with a typical resolution of $10 \%$. The DNA is fluorescently stained with the dye YOYO-1, at a stoichiometry of one dye molecule per 7.5 base pairs. The fluorescent signal is imaged onto an avalanche photodiode detector with a high numerical aperture objective. The signal is strong enough that photon counting detection is not required, an advantage over traditional flow cytometric measurements.

By designing a junction in the channel, molecules and cells can be sorted. After measuring the length of the molecule, a servo can decide to "keep" or "discard" the cell. We have demonstrated sorting of fluorescent beads and $E$. Coli cells in our devices. Manipulation was accomplished with electro-osmotic flow. Electrodes were inserted at the ends of the three channels, and a potential of up to $60 \mathrm{~V}$ was used to manipulate the cells and beads. We were able to move cells at speeds of 100 microns $/ \mathrm{sec}$, and have demonstrated switching times of $\sim 75$ milliseconds.

\section{DEVICE FABRICATION AND MOUNTING}

Negative master devices were fabricated in silicon, and used as molds for the silicone elastomer. Standard contact photolithography techniques were used to pattern the oxide surface of a silicon wafer, which was then etched by reactive ion etch with a $\mathrm{C}_{2} \mathrm{~F}_{6} / \mathrm{CHF}_{3}$ gas mixture. A 3 minute $\mathrm{O}_{2} \mathrm{RIE}$ was used to remove carbon residue on the silicon surface. The oxide was then used as a mask for the silicon underneath, which was etched with $\mathrm{KOH}$. The silicone elastomer (General Electric RTV 615) components were mixed together, and pumped in an evacuated chamber for 30 minutes to remove dissolved oxygen. The liquid elastomer was then poured on the mold (spin coating was used in cases where the device thickneess needed to be well controlled) and cured in an oven at 95 degrees for two hours. After this, the devices could be peeled from the silicon master and would bond hermetically to glass.

The devices were patterned as shown in figure 1 . The large channels had lateral dimensions of 100 microns, which narrowed down to 5 microns at the $T$ junction. The depth of the channels ranged from 2.5 to 20 microns. In early prototypes, we found that due to the large aspect ratio ( 100 microns in width by 2 microns in depth), some of the elastomer channels would bow and and block themselves by sealing directly to the glass. This was remedied in later versions by adding support pillars to the mask that would prop up the large channels and prevent bowing (see figure 1).

The elastomer is naturally hydrophobic, preventing aqueous solution from entering the channels. We modified the surface of the devices by soaking it in dilute $\mathrm{HCl}(0.02 \%$ in water $)$ for 40 minutes at $43 \mathrm{C}$. After this treatment, the devices were hydrophilic and aqueous solution would easily enter by capillary 
action. The devices could be cleaned and reused several times if desired.

Devices were mounted in one of two ways. For the DNA sizing devices, sample wells were created during the fabrication process by gluing small aluminum cylinders to the silicon wafer molds. The final RTV devices were attached to a cover slip in such a way that the sample wells were only partially covered. This allowed access with a pipette tip for introduction of the sample. The flow rate was determined by capillary action within the channels.

For the cell sorting devices, an inverted method was used. Holes were cut in the devices with a razor blade at the input and output portions, forming sample wells. The device was placed on a cover slip or microscope slide, and used in an inverted microscope. Then the aqueous samples were introduced via capillary action, and controlled with clectro-osmotic forces. Tin plated copper electrodes were inserted into sample wells for this purpose.
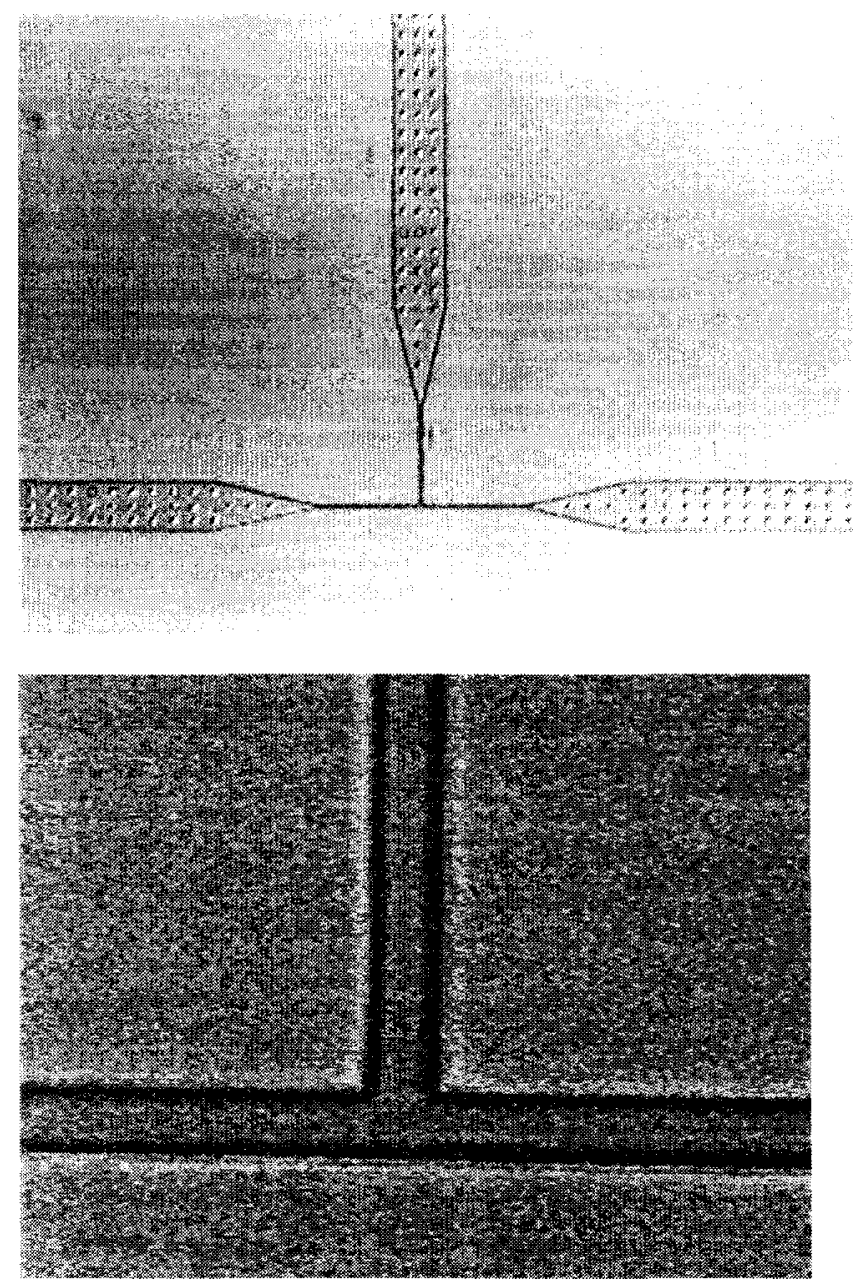

Figure 1. Top: Optical micrograph of an elastomer device. The large channels are 100 microns wide; the channels narrow down to a width of 5 microns at the T-junction. The depth of the channels is 3 microns. The large channels extend approximately 1 $\mathrm{cm}$ in length (not shown) for making macroscopic fluidic contacts. Bottom: Higher magnification image of the T-junction. The channels here are 5 microns wide. Note the high fidelity of the replica fabrication process for the straight lines and corners of the channels.

\section{EXPERIMENTAL SETUP}

For the DNA sorting devices, an upright optical microscope (Olympus) was used. A $3 \mathrm{~mW}$ air cooled argon ion laser (Uniphase) emitting at $488 \mathrm{~nm}$ was used for fluorescent excitation. The laser was focused through a $60 \times 1.4 \mathrm{NA}$ oil immersion objective, which was also used to collect the emitted fluorescence. Auxiliary lenses were used to adjust the sıze of the focused spot to approximately 30 microns. The large spot size was chosen to give uniform excitation across the width of the channel. The quality and uniformity of the spot was evaluated by imaging a thin layer of fluorescein in solution with a CCD camera. The image was digitized and evaluated for symmetry and gaussian shape. Dielectric filters were used to filter laser tube fluorescence (CVI $488 \mathrm{~nm}$ line filter) and to reduce background and scattered light from the emitted fluorescence (Chroma D535/50M). A dichroic filter was used to introduce the laser light into the optıcal train (Chroma 500 DCLP).

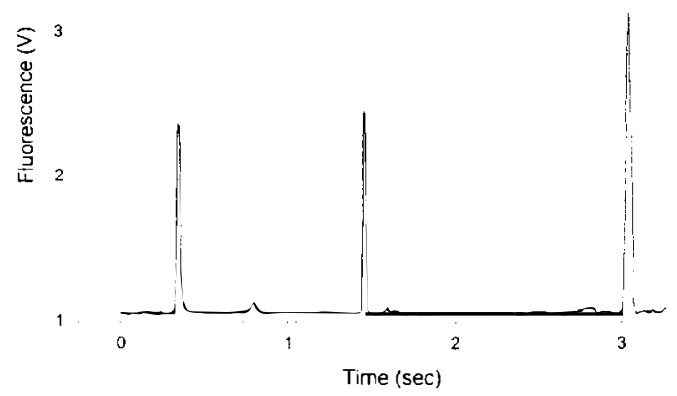

Figure 2: Pulses from single molecules of fluorescently stained DNA as they flow through the device.

Fluorescence was imaged onto a $5 \mathrm{~mm}$ avalanche photodiode detector (Advanced Photonics). The detector was cooled to -40 degrees with a two stage thermoelectric cooler (ITI $6320 / 157 / 040 \mathrm{C}$ ), which reduced the dark current of the detector from $50 \mathrm{nA}$ to $90 \mathrm{pA}$. The detector was biased at $2400 \mathrm{~V}$, giving an estimated gain of 500-1000. The photocurrent was converted to a voltage by a Burr Brown OPI 28 ultra low noise op amp operating at a gain of $100 \mathrm{mV} / \mathrm{nA}$. A second stage amplifier provided additional voltage gain of 10 . The signal was low pass filtered at $1.6 \mathrm{kHz}$, and digitized at $5 \mathrm{kHz}$ by a National Intruments Lab PC1200 board on a PC running Labview. Analysis of the noise level as a function of laser power indicates that the background fluctuations are shot noise limited.

The depth of focus of the microscope was checked by centering a 1 micron fluorescent bead in the laser beam. The detector output as a function of focal distance shows that the signal is essentially flat over a depth of 5 microns. This was then used to design the microfabricated devices; the depth was chosen so that the DNA molecules always remained in the plane of focus of the microscope. 
Lambda phage DNA (Gibco) was either digested with HindIII or ligated, then was diluted in buffer (TE with $10 \mathrm{mM}$ $\mathrm{NaCl}$ ) and stained with the intercalating dye YOYO-1 (Molecular Probes) at a stoichiometry of approximately one dye molecule per 7.5 base pairs. Single molecules of DNA gave measurable pulses whose height corresponded to the length of the molecule. Pulses were collected in large batches and then analyzed off line with home written software for peak detection.

The total interaction region imaged on the detector is approximately 375 femtoliters, smaller than is achievable with standard methods of flow cytometry. This volume is largely determined by the size of the laser spot, and can be reduced further by the use of cylindrical optics.

The cell sorting devices were mounted on an inverted microscope (Zeiss). Electrodes were attached as described above, and a switching box was built to manipulate a potential of 60 Volts between the three channels. Each channel could be independently set for either $60 \mathrm{~V}$, ground, or float. E. Coli cells expressing green fluorescent protein were introduced into the devices by capillary action and observed with fluorescence microscopy. Fluorescence was excited with a mercury lamp, and recorded on videotape with a CCD camera (Hamamatsu). The video images were later digitized and the position of the cells as a function of time was measured.

\section{RESULTS}

Data was taken from two heterogenous solutions of DNA. In the first, a $\lambda$ HindIII digest, fragments of length $2 \mathrm{kbp}-$ $23 \mathrm{kbp}$ were identified. The typical resolution was $10 \%$ per molecule. A histogram of the peak heights is shown in figure 3. By locating the center of the peaks in the histogram, the cumulative precision is higher. 977 molecules were analyzed. The smallest resolved molecule is on the order of of 2 kilobase pairs. This is not an intrinsic limit of the method, and we believe that with further improvements to the electronics and analysis we can improve the sensitivity to 1 kilobase pair. We intend to continue improving the fluidic portion of the device to increase the throughput.

It is worth pointing out the extremely small amount of material used for such an analysis. A total of 977 molecules were analyzed, i.e. on the order of a zeptomole. The analysis was performed in a total reagent volume of tens of nanoliters. Again, these are not intrinsic limits of the system and we believe that with further improvements, the reaction volumes can be reduced substantially.

The second DNA sample we analyzed was a $\lambda$ ladder; peaks ranging from $50 \mathrm{kbp}$ to $200 \mathrm{kbp}$ were identified. 1761 molecules were identified in 25 minutes of running time. The resolution per molecule is about $5 \%$. There is a clear trend over the data sets showing improving resolution with increasing molecule length. This particular length range of DNA molecules is impossible to separate with ordinary gel electrophoresis; one must resort to pulsed field gels.
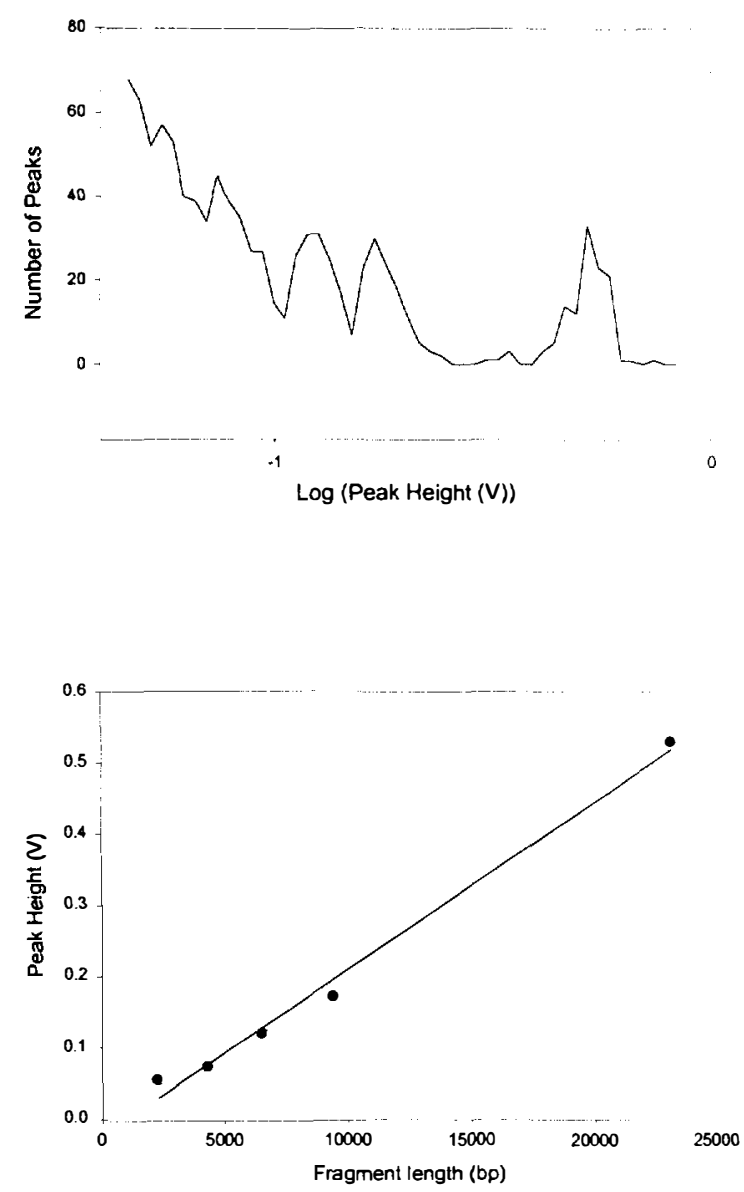

Figure 3: Top: A histogram of pulse heights from the DHindIII digest. The heights were measured by taking the maximum value of the peaks; no fitting was done. Fragments of length $2 k b p, 4 k b p$, $6 \mathrm{kbp}, 9 \mathrm{kbp}$ and $23 \mathrm{kbp}$ were identified. Bottom: The sizes of the fragments are known independently of this measurement, and can be used to test the precision of the method. The peak height versus length is plotted, showing good linear agreement.

We have also demonstrated the principle of cell sorting with electro-osmotic manipulation in our devices. E. Coli cells were manipulated with electric fields, and switched between one of two possible output channels. A graph of the position versus time of a single $E$. Coli cell as the field is repeatedly reversed is shown in figure 5 . The estimated switching time is $50-100$ milliseconds, leading to a maximum cell sorting rate of $10-20 \mathrm{~Hz}$ We believe with higher potentials and some device design improvements, we could reach a single channel switching time of 1 millisecond. Then with 100 channels switching in parallel, the overall throughput of the device would be $100 \mathrm{kHz}$. 

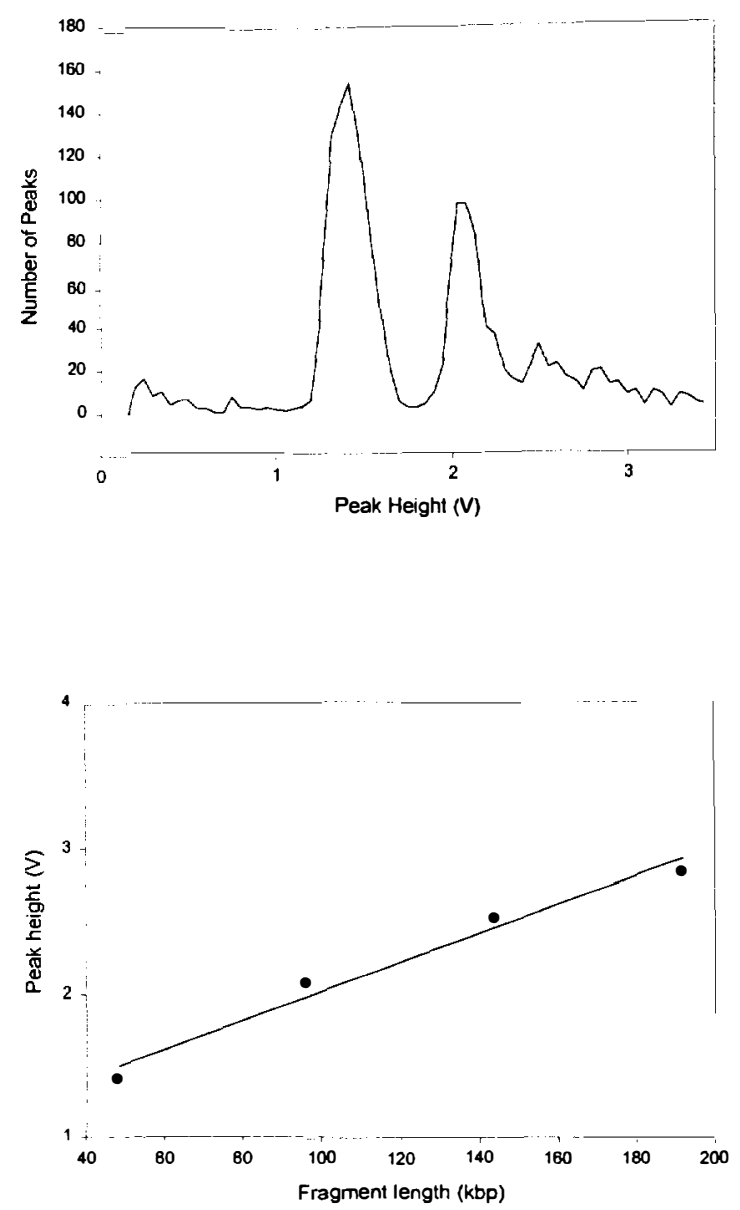

Figure 4: Top: A histogram of pulse heights from the $\lambda$ ladder. The first through third multimers can clearly be seen, while the fourth multimer is barely resolved. Bottom: Graph of known fragment length versus peak position.

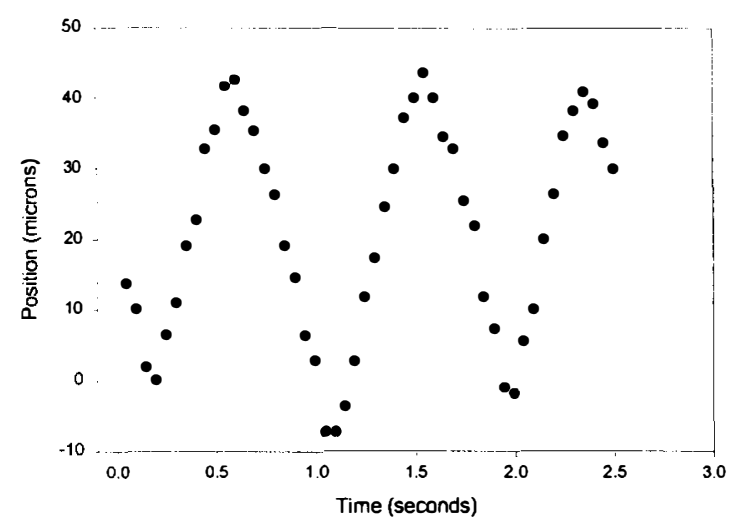

Figure 5: E Coli position versus time as a $60 \mathrm{~V}$ potential is repeatedly reversed.
One concern is whether the high electric fields in the device would damage or kill the bacteria. To test this, we drove bacteria through the devices and collected them at the output port with a micropipette. The bacteria were then grown up an agar plates, showing that they could indeed survive the sorting process.

\section{CONCLUSION}

We have successfully demonstrated DNA sizing and cell sorting in microfabricated devices. With further improvements, we believe that this method will be competitive with the current state of the art in gel electrophoresis, and will find specialized applications in cell sorting, such as lab on a chip. These devices are extremely easy to fabricate from a silicon master, reducing production costs and rendering the devices truly disposable. This is advantageous for cell sorting applications in which contamination is a concern.

There are numerous possible applications for such technology. We anticipate that the DNA analytical system will be used for many routine biological assays, due to its speed and low material requirement. One possible application is DNA fingerprinting. A portable system could be brought to the scene of a crime for direct analysis on small blood samples. Since the system does not require PCR, the chance of false signals is reduced. Furthermore, the chance of laboratory error due to contamination and handling will be reduced.

We envision both technologies demonstrated in this paper as stepping stones to building gene cloning "lab on a chip" devices. Small amounts of DNA can be recovered from restriction and ligation reactions, and separated according to size.

Performing transfection on the chip allows very small (nanoliter) reaction volumes, so only small amounts of DNA are necessary. The properly transfected cells can then be separated via fluorescent or colorimetric assays in the cell sorting device. Single cells can then be grown into macroscopic amounts necessary for clone libraries, etc.

\section{REFERENCES}

[1] A. Castro, F.R. Fairfield and E.B. Schera, Anal. Chem. 65, 849 (1993).

[2] Z Huang et al, Nucleic Acids Res. 24, 4202 (1996).

[3] R.H. Carlson et al, Phys. Rev. Lett. 79, 2149 (1997).

[4] R.J. Jackson, J.L. Wilbur and G.M. Whitesides, Science 269, 664 (1995).

[5] H.P. Chou, C. Spence, A. Scherer and S. Quake, to appear in Proceedings of the SPIE (1998).

- To whom correspondence should be addressed. Email quake@cco.caltech.edu, fax (626) 7938675

\section{ACKNOWLEDGEMENTS}

We thank Frances Arnold for the generous loan of equipment used in the cell sorting device. 